\title{
Substanzkonsum, Störungen durch Substanzkonsum und begleitende psychische Störungen bei Jugendlichen
}

\section{Zahlen aus einer Spezialambulanz für Suchterkrankungen}

\author{
Melina Wiedmann ${ }^{1}$, Josefine Atzendorf ${ }^{2}$, Lukas Andreas Basedow ${ }^{1}$, Veit Roessner ${ }^{1}$, \\ Yulia Golub ${ }^{1}$ und Sören Kuitunen-Paul ${ }^{1}$
}

\author{
Klinik und Poliklinik für Kinder- und Jugendpsychiatrie und -psychotherapie, Medizinische Fakultät, Technische Universität Dresden, \\ Dresden \\ Munich Center for the Economics of Aging (MEA), Max-Plank-Institut für Sozialrecht und Sozialpolitik, München
}

\begin{abstract}
Zusammenfassung: Fragestellung: Nur wenige Kliniken für Kinder- und Jugendpsychiatrie (KJP) bieten eine ambulante Behandlung von Jugendlichen mit Substanzkonsumstörungen (SUDs) an. Daher fehlen Angaben, welche Konsummuster, SUDs und begleitenden psychischen Störungen diese Jugendlichen aufweisen. Methodik: N = 201 Patient_innen einer universitären Spezialambulanz (12-19 Jahre alt) wurden bezüglich Konsummustern, SUDs und aktuellen psychischen Störungen mittels Interview untersucht. Es wurden diesbezüglich deskriptive Darstellungen zu Prävalenzzahlen der SUDs, Konsummustern und begleitenden psychischen Störungen aufgeteilt nach Geschlecht und aktuellem Alter erstellt. Ergebnisse: Tabak (88\%) und Cannabis (86\%) waren die verbreitetsten Substanzen. Bei $67 \%$ aller Patient_innen wurde mehr als eine SUD festgestellt. SUDs bezogen sich am häufigsten auf Cannabis ( $84 \%)$, gefolgt von Tabak (77\%). $72 \%$ aller Patient_innen zeigten eine die SUD begleitende psychische Störung, insbesondere Störungen des Sozialverhaltens (40\%), hyperkinetische Störungen (21\%) und depressive Störungen (18\%). Schlussfolgerungen: Ambulant behandelte jugendliche KJP-Patient_innen mit SUDs präsentieren sich häufig mit begleitenden psychischen Störungen. Angebotene Behandlungsprogramme sollten in der Behandlung von SUDs insbesondere Störungen des Sozialverhaltens, Depressionen und hyperkinetische Störungen berücksichtigen.
\end{abstract}

Schlüsselwörter: Adoleszenz, Affektive Störungen, Störungen des Sozialverhaltens, Substanzkonsumstörungen, Sucht

Substance Use, Resulting Disorders, and Collateral Mental Disorders Among Adolescents in a Special Outpatient Institutions for Addictions

Abstract: Objective: Only few clinics offer the outpatient treatment of substance use disorders (SUDs) among adolescents. Therefore, only limited data describe substance use patterns, SUDs, and co-occurring psychiatric disorders characteristic of adolescents who present in such outpatient clinics specialized in the treatment of SUDs. Method: Via interview we collected data from $n=201$ patients between 12 and 19 years concerning their substance use, SUDs, and current co-occurring psychiatric disorders. We created descriptive presentation of data regarding use patterns, SUDs, and co-occurring disorders divided by sex and current age. Results: Tobacco (88\%) and cannabis (86\%) were the most frequently used substances. $67 \%$ of all patients presented with more than one SUD, cannabis use disorder being the most prevalent one (84\%). $72 \%$ presented with at least one co-occurring disorder, with conduct disorders (40\%), attention deficit (hyperactivity) disorders (21\%), and depressive disorders (18\%) being the most frequent ones. Conclusions: Adolescent SUD patients often present with co-occurring psychiatric disorders. Institutions for adolescent SUD treatment should also focus on treating co-occurring conduct disorders, depression, and attention deficit disorders.

Keywords: Substance use disorders, adolescence, affective disorders, conduct disorders, addiction 


\section{Einleitung}

Weltweit sind Störungen durch den Konsum psychoaktiver Substanzen oder Substanzkonsumstörungen (SUDs) eines der größten Gesundheitsrisiken für junge Menschen (Mokdad et al., 2016). Konsumprävalenzen für psychoaktive Substanzen in Deutschland sind hoch und teils über dem europäischen Durchschnitt, z. B. für Alkohol mit 37\% der Jugendlichen in den letzten 30 Tagen (Orth \& Merkel, 2019), für Cannabis mit einer Lebenszeitprävalenz von $10 \%$ und für sonstige illegale Drogen mit einer Lebenszeitprävalenz von 2\% (Orth \& Merkel, 2020). Dabei stellen in Deutschland neue psychoaktive Substanzen sowie Methamphetamin die zweithäufigsten illegalen Substanzen nach Cannabis dar (Gomes de Matos, Atzendorf, Kraus \& Piontek, 2016). In Bezug auf Methamphetamin zeigen sich deutschlandweit deutliche regionale Unterschiede, wobei Sachsen und Thüringen die höchsten Lebenszeitprävalenzen aufweisen (Gomes de Matos, Hannemann, Atzendorf, Kraus \& Piontek, 2018). Aus dem Substanzkonsum entwickeln sich in zahlreichen Fällen SUDs (Behrendt et al., 2008; Choi, Lu, Schulte \& Temple, 2018; Kipping, Campbell, MacArthur, Gunnell \& Hickman, 2012), was in Deutschland auf 2 bis $3 \%$ der Jugendlichen und jungen Erwachsenen bzgl. Betäubungsmittel zutrifft (Beesdo-Baum et al., 2015; Lieb et al., 2000), auf 6\% bzgl. Alkohol (Beesdo-Baum et al., 2015) und auf $18 \%$ bzgl. Tabak (Lieb et al., 2000). Cannabiskonsumstörungen bilden den Hauptvorstellungsgrund für Jugendliche, die eine ambulante bzw. stationäre suchttherapeutische Behandlung aufsuchen oder aufsuchen müssen (Thaller et al., 2016; Thomasius et al., 2020). Bei Jugendlichen mit SUDs ist ein deutlicher Anstieg der Behandlungsinanspruchnahme erkennbar. Entgegen dem $10 \%$ igen Rückgang an Behandlungen in Notaufnahmen in sächsischen Akutkrankenhäusern in den Jahren 2012 bis 2016 stieg die Anzahl an Behandlungen bei den unter 15-Jährigen um $38 \%$ (Sächsisches Staatsministerium für Soziales und Gesellschaftlichen Zusammenhalt, 2019).

Einer SUD bei Jugendlichen geht zudem häufig eine weitere psychische Störung voraus (Holtmann et al., 2011), sodass SUDs in diesen Fällen als sekundäre Störungen angesehen werden können. Bei jugendlichen Patient_innen mit SUDs dominieren unter den begleitenden Störungen die (kombinierten) Störungen des Sozialverhaltens (Prävalenz 50 bis $74 \%$; Diamond et al., 2006; Hser, Grella, Collins \& Teruya, 2003), Aufmerksamkeitsdefizit-/Hyperaktivitätsstörungen ([ADHS] Prävalenz 13 bis $77 \%$; Chan, Dennis \& Funk, 2008; Diamond et al., 2006; Grella, Joshi \& Hser, 2004), gefolgt von depressiven Störungen (Prävalenz 14 bis $50 \%$; Bukstein, Glancy \& Kaminer, 1992; Diamond et al., 2006; Grella, Hser, Joshi \& Rounds-Bryant, 2001) und Angststörungen
(Prävalenz 7 bis 40\%; Chan et al., 2008; Diamond et al., 2006; Kaminer, 1996). Die Schwankungen in der Prävalenz sind mitunter auf unterschiedliche Behandlungssettings (ambulant/stationär) zurückzuführen. Diese hohen Raten an begleitenden psychischen Störungen bei SUDs werden auch in nichtklinischen Studien gefunden, wonach im Schnitt mehr als die Hälfte aller Jugendlichen mit SUDs eine begleitende psychische Störung aufweist (Armstrong \& Costello, 2002). Liegen begleitende Störungen vor, so sind die Chancen auf eine erfolgreiche Behandlung deutlich niedriger und das Risiko steigt, zukünftig eine erneute SUD-Behandlung in Anspruch zu nehmen (Chen, Strain, Crum \& Mojtabai, 2013; Harris \& Edlund, 2005). In Deutschland gibt es, im Gegensatz zu anderen europäischen Ländern (z.B. Norwegen, Schweden, Dänemark), nur wenige Einrichtungen, die auf die parallele Behandlung von SUDs und begleitenden psychischen Störungen spezialisiert sind (European Monitoring Centre for Drugs and Drug Addiction, 2015) - obwohl eine parallele Behandlung größere Behandlungserfolge erzielt (Loeber et al., 2011). Außerdem fehlen ambulante Behandlungskonzepte, die laut Stepped-Care-Ansatz ein Bindeglied zwischen niedrigschwelliger Beratung und hochschwelliger stationärer Behandlung darstellen sollten (Bühringer \& Rumpf, 2018; Rumpf et al., 2017) und zudem rückfallpräventiv angeboten werden können.

Um neu entstehende ambulante Strukturen auf zukünftige Patient_innen zuzuschneiden, muss das ambulante Behandlungsangebot auf deren spezifische Konsumpräferenzen, SUD-Kombinationen und die häufigsten begleitenden psychischen Störungen abgestimmt sein. Dafür sollen hier erste Daten aus einer ambulanten Inanspruchnahmestichprobe präsentiert werden. Angesichts der in Deutschland bestehenden Unterversorgung von Kindern und Jugendlichen mit SUDs (Thomasius et al., 2020) ist dies besonders wichtig, um effiziente und nachhaltige Behandlungserfolge zu erzielen.

\section{Methodik}

\section{Studiendesign}

An der Klinik für Kinder- und Jugendpsychiatrie und Psychotherapie des Universitätsklinikums C.G. Carus Dresden besteht seit 2017 eine Spezialambulanz für Suchterkrankungen. Die Inanspruchnahmepopulation dieser Ambulanz wurde für den Zeitraum seit Beginn der Erfassung von personenbezogenen Daten (November 2017 bis November 2020) untersucht. Jugendliche und deren gesetzliche Vertreter_innen, die sich in der Spezialambulanz 
vorstellten, wurden während ihres ersten Termins über eine Studie, die ein neu entwickeltes Gruppentherapieprogramm für die ambulante Behandlung von SUDs bei Jugendlichen (DELTA; Golub, Basedow, Meiron-Zwipp, Kuitunen-Paul \& Roessner, 2021) evaluiert und Teil der Regelbehandlung ist, aufgeklärt. Alle Prozesse zur Datenerhebung wurden in die üblichen diagnostischen Prozesse der ambulanten Behandlung eingebettet. Soziodemografische Daten, Angaben zum Substanzkonsum und ein strukturiertes, klinisch-diagnostisches Interview wurden von klinisch geschulten Mitarbeiter_innen durchgeführt, bevor irgendeine Intervention begann. Es wurden ausschließlich Querschnittsdaten für die vorliegende Auswertung verwendet. Studienteilnehmer_innen erhielten $20 €$ als Aufwandsentschädigung für die Teilnahme an weiteren Terminen der Studie, welche in der vorliegenden Analyse nicht berücksichtigt wurden. Alle Studienabläufe wurden in Übereinstimmung mit der Deklaration von Helsinki durchgeführt und durch den Prüfungsausschuss der Universitätsklinik C.G. Carus Dresden (EK 66022018) bestätigt und unter https://clinicaltrials.gov (NCT03444974) registriert.

\section{Proband_innen}

Von $n=237$ Jugendlichen, die sich im Erhebungszeitraum vorstellten, gaben $n=201$ (85\%) Jugendliche und deren gesetzliche Vertreter_innen ihr freiwilliges, schriftliches Einverständnis zur Auswertung ihrer Daten zu wissenschaftlichen Zwecken. Das Einverständnis beinhaltet vor allem freiwillige Vorstellungen, vereinzelt aber auch Vorstellungen im Rahmen einer polizeilichen Vorführung zur Fallkonferenz (nach Erteilung einer polizeilichen Zuführung durch den oder die Jugendrichter_in).

\section{Instrumente}

\section{Aktuelle psychische Störungen nach ICD-10}

Die Diagnosen aktueller psychischer Störungen wurden im Rahmen des Erstgesprächs durch Mitarbeiter_innen der Spezialambulanz für Suchterkrankungen (Ärzt_innen in Weiterbildung, Psychotherapeut_innen, Psycholog_innen) erhoben. Die diagnostizierten SUDs beinhalteten psychische und Verhaltensstörungen durch Alkohol (F10.X), Opioide (F11.X), Cannabinoide (F12.X), Sedativa oder Hypnotika (F13.X), Kokain (F14.X), andere Stimulanzien, einschließlich Koffein (F15.X), Halluzinogene (F16.X), Tabak (F17.X), flüchtige Lösungsmittel (F18.X) und multiplen Substanzgebrauch sowie Konsum anderer psychotroper Substanzen (F19.X). Für die vorliegende Analyse wurden lediglich die Diagnosen F1X.1 Schädli- cher Gebrauch und F1X.2 Abhängigkeit ausgewertet. Anhand eines strukturierten Leitfadens wurden alle sechs Symptome einer SUD erhoben und Diagnosen für schädlichen Gebrauch (weniger als drei Symptome) und für Abhängigkeit (drei oder mehr erfüllte Symptome) gestellt. Weiterhin wurden für die vorliegende Beschreibung der Stichprobe diagnostizierte Störungen entsprechend den folgenden Störungsgruppen zusammengefasst: SUDs (F1X.1 und F1X.2), depressive Störungen (F32.X, F33.X), Angststörungen (F40.X, F41.X), Posttraumatische Belastungsstörung (PTBS; F43.1), hyperkinetische Störungen (F90.X, F98.8), Störung des Sozialverhaltens (F90.1, F91.X und F92.X), Persönlichkeitsentwicklungsstörungen (F93.8 und F43.X außer F43.1) und psychotische Störungen (F1X.5, F1X.7, F30.2, F2X.X).

\section{Soziodemografische Daten}

Die gesetzlichen Vertreter_innen der Jugendlichen gaben mittels Fragebogen Auskunft über die soziodemografischen Daten, z. B. „Wie verlief die bisherige schulische Laufbahn?" (Antwortmöglichkeiten: „regelgerecht", „wiederholt“, „Schulabbruch“), oder „Gab es bei Ihnen in Bezug auf Ihr Kind bereits eine Vorbehandlung oder Inanspruchnahme einer Beratung, welche die Vorstellung in unserer Ambulanz empfohlen hat? Wenn ja, welche waren das?" (Antworten wurden in die Kategorien Jugendamt, Beratungsstelle, Kinder- und jugendpsychiatrische Einrichtung, Therapeutische/pädagogische Wohngruppe, Ohne Vorbehandlung zusammengefasst).

\section{Körperliche Merkmale}

Im Rahmen einer körperlichen Untersuchung wurden von den Patient_innen Daten zu nichtsuizidalem selbstverletzendem Verhalten (NSSV) erfasst sowie Größe und Gewicht gemessen. Für die Erhebung von Daten zu NSSV wurden die Patient_innen gefragt, ob sie sich jemals in ihrem Leben in selbstschädigender Weise ohne Suizidabsicht selbst verletzt hatten (schneiden, brennen, gegen Gegenstände schlagen), sowie körperliche Folgeerscheinungen (Narben) dokumentiert.

\section{Substanzkonsum}

Alle Proband_innen wurden während ihres ersten Termins über die Häufigkeit ihres Konsums und die durchschnittliche Menge pro Konsumtag der folgenden Substanzen für den Zeitraum des letzten Monats und des letzten Jahres befragt: Tabak, Alkohol, Cannabis, MDMA, Amphetamin, Methamphetamin, Kokain, Halluzinogene, Ketamin, Opiate und andere Substanzen (,An wie vielen Tagen pro Monat hast du die Substanz durchschnittlich über das letzte Jahr konsumiert?" oder „Wie viel von der Substanz hast du im letzten Jahr an einem durchschnittlichem Konsumtag zu dir genommen?"). 


\section{Statistische Auswertung}

Alle statistischen Auswertungen wurden mit IBM SPSS Statistics 27.0 (IBM SPSS Statistics for Windows, Version 27.0) durchgeführt. Für die Auswertung der soziodemografischen Daten wurden Chi-Quadrat-Tests bezüglich der Faktoren Geschlecht (Mädchen/Jungen) und Altersklasse (Mediansplit: $</ \geq 16$ Jahre) mit Bonferroni-korrigiertem Signifikanzniveau von $p<.0014$ (.05 geteilt durch 36 Vergleiche) berechnet, um für eine Alpha-Fehler-Kumulierung zu kontrollieren. Fehlende Werte bei Items in einzelnen Fragebögen wurden von den Analysen listenweise ausgeschlossen und es wurden die Angaben zu gültigen Prozent verwendet.

Für die Berechnung zu Unterschieden in den Angaben zum Substanzkonsum (abhängige Variablen) zwischen den Prädiktoren Geschlecht (Mädchen/Jungen) und Altersklasse $(</ \geq 16$-Jährige) wurden univariate Varianzanalysen herangezogen. Für die Auswertung der SUDs und begleitenden Störungen wurden binomiale logistische Regressionsanalysen mit den Faktoren Geschlecht und Altersklasse als Outcome gerechnet. Das Signifikanzlevel wurde in den Berechnungen zu ANOVAS und binomialen Regressionen als $p<.05$ (zweiseitig) definiert. Die Effektgrößen wurden nach der Definition von Cohen (Cohen, 2009) in kleine Effekte $\left(|r| \geq .10,\left|\eta_{\text {part }}^{2}\right| \geq .01,|\varphi| \geq .10\right)$, mittlere Effekte $\left(|r| \geq .30,\left|\eta_{\text {part }}^{2}\right| \geq .06,|\varphi| \geq .30\right)$ und große Effekte $\left(|r| \geq .50,\left|\eta_{\text {part }}^{2}\right| \geq .14,|\varphi| \geq .50\right)$ unterteilt.

\section{Ergebnisse}

\section{Soziodemografische Daten}

Das Durchschnittsalter lag bei 16.0 Jahren $(S D=1.3)$, wobei Jungen älter waren als Mädchen $\left(M_{\text {männlich }}=16.2, S D_{\text {mӓnn- }}\right.$ lich $=1.2$ vs. $\left.M_{\text {weiblich }}=15.6, S D_{\text {weiblich }}=1.4\right)$. Die Jugendlichen lebten nur zu einem geringen Anteil mit beiden leiblichen Elternteilen zusammen (16\%). $10 \%$ aller Jugendlichen gaben an, die Schule abgebrochen zu haben. Mit $18 \%$ aller Patient_innen stellte sich fast jede/jeder Fünfte in der Ambulanz allein auf Veranlassung der Eltern - ohne Vorbehandlung oder Empfehlung einer Beratungsstelle oder des Jugendamts - vor. Jede/r zweite Patient_in (51\%) gab eine kinder- und jugendpsychiatrische Vorbehandlung an (siehe Tabelle 1).

\section{Substanzkonsum}

Laut Selbstauskunft waren Tabak $(88 \%$ mindestens einmal im letzten Jahr) und Cannabis (86\% mindestens einmal im letzten Jahr) die verbreitetsten Substanzen, die im letzten Jahr und im letzten Monat konsumiert wurden (siehe Tabelle 2 und elektronisches Supplement [ESM] 1, Tabelle). Tabak wurde häufiger konsumiert ( $M=26.5$ Konsumtage im letzten Jahr, $S D=7.5)$ als Cannabis $(M=16.3$ Konsumtage im letzten Jahr, $S D=11.5$ ). Alkohol wurde von $70 \%$ mindestens einmal pro Monat im letzten Jahr konsumiert mit $M=7.6$ Konsumtagen pro Monat, $S D=9.1$. Fast jede/r dritte Patient_in (31\%) berichtete, im letzten Jahr mindestens einmal pro Monat MDMA konsumiert zu haben, mit im Schnitt wöchentlichem Konsum ( $M=5.6$ Tage, $S D=7.1)$. Amphetamine und Methamphetamin wurden von $16 \%$ mindestens einmal pro Monat im letzten Jahr konsumiert mit $M_{\text {Amphetamine }}=6.3$ Konsumtagen pro Monat, $S D_{\text {Amphetamine }}=8.0$ und $M_{\text {Methamphatemin }}=7.6$ Konsumtage pro Monat, $S D_{\text {Methamphatemin }}=8.6$.

Tabak wurde unter allen Substanzen mit $M=12.7$ Jahren $(S D=2.0)$ am frühesten erstmalig konsumiert (siehe ESM 2, Tabelle), gefolgt von Alkohol $(M=13.3, S D=1.5)$ und Cannabis $(M=13.7, S D=1.4)$. Patient_innen, die bei Erstvorstellung älter als 16 Jahre waren, berichteten ein höheres Erstkonsumalter - ein Unterschied, der in Bezug auf Tabak, Alkohol, Cannabis, Ecstasy/MDMA, Amphetamine und Methamphetamin signifikant war $(F[1,13] \geq 7.29$, $p \leq .018, \eta^{2} \geq .22$ ).

Mädchen berichteten, tendenziell an mehr Tagen im letzten Jahr MDMA konsumiert $\mathrm{zu}$ haben als Jungen $\left(F[1,28]=3.20 ; p=.084 ; \eta^{2}=.10 ;\right.$ siehe Tabelle 2$)$. Methamphetamin wurde während des letzten Monats tendenziell an mehr Tagen von den über 16-Jährigen Patient_innen konsumiert $\left(F[1,4]=1.76, p=.255, \eta^{2}=.31\right.$; siehe ESM 3, Tabelle). Unter denjenigen, die mindestens einmal pro Monat im letzten Jahr die jeweilige Substanz konsumierten, berichteten Mädchen deutlich mehr Konsumtage im letzten Jahr als Jungen - sowohl bzgl. Methamphetamin $\left(F[1,13]=1.48, p=.246, \eta^{2}=.10\right)$ als auch bzgl. Kokain $\left(F[1,6]=1.34, p=.291, \eta^{2}=.18\right.$; siehe Tabelle 2).

\section{SUDs}

$67 \%$ aller Patient_innen zeigten eine SUD bzgl. mehr als einer Substanz (siehe Tabelle 3) - bzw. 77\% bei Einbezug von Tabakkonsumstörungen, die aber nie Vorstellungsgrund waren. Mit $84 \%$ wurde beim Großteil aller Patient_innen eine psychische und Verhaltensstörung durch Cannabinoide festgestellt, verteilt auf $40 \%$ mit Cannabisabhängigkeit und $44 \%$ mit schädlichem Cannabisgebrauch (siehe Abbildung 1). Die zweithäufigste Diagnose bildeten psychische und Verhaltensstörungen durch Tabak (77\%). Psychische und Verhaltensstörungen durch Alkohol (52\%) und Stimulanzien (45\%) wurden bei fast jeder/m zweiten Patient_in diagnostiziert. Hier überwog der Anteil derjenigen mit schädlichem Gebrauch (41\% für Alkohol bzw. 30 \% für Am- 


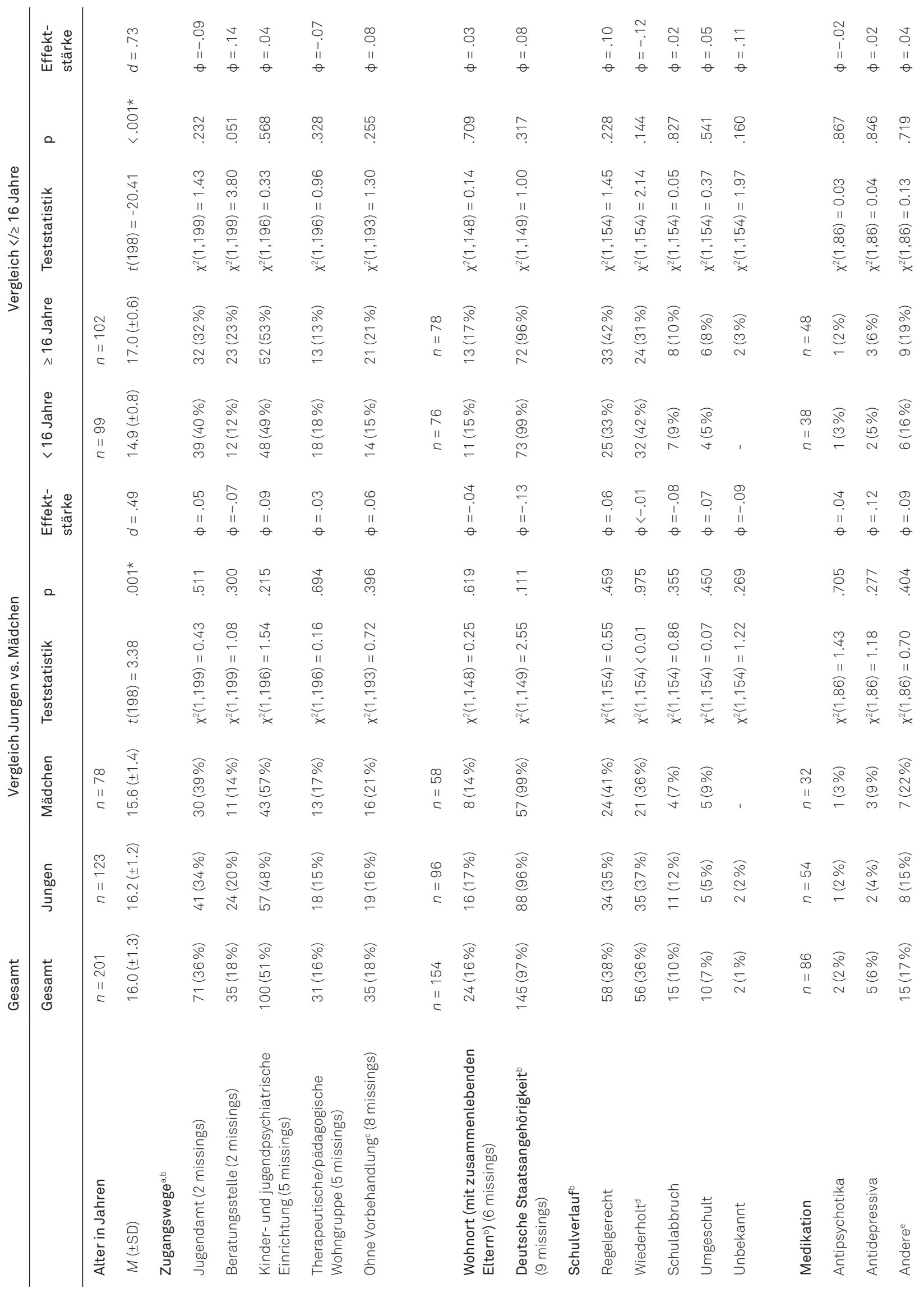




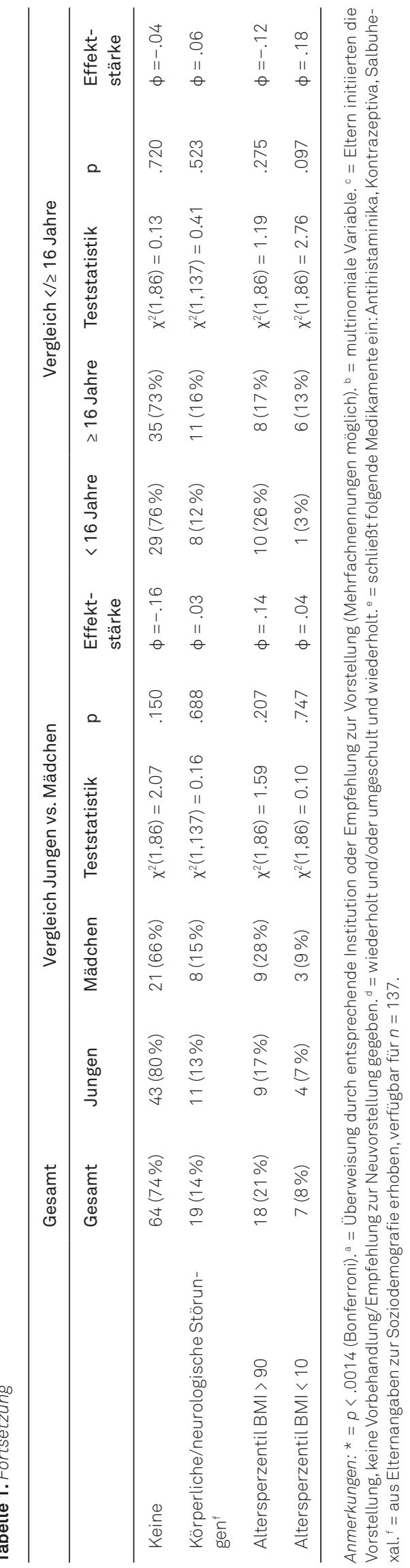

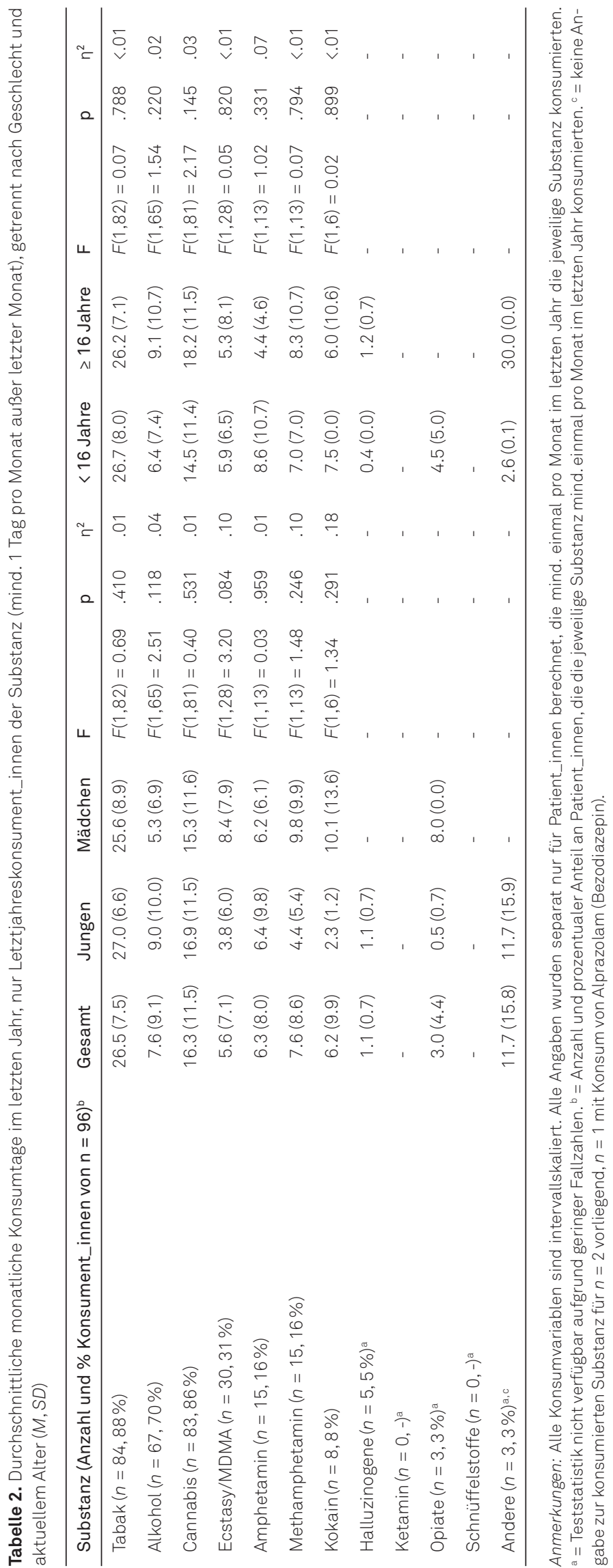




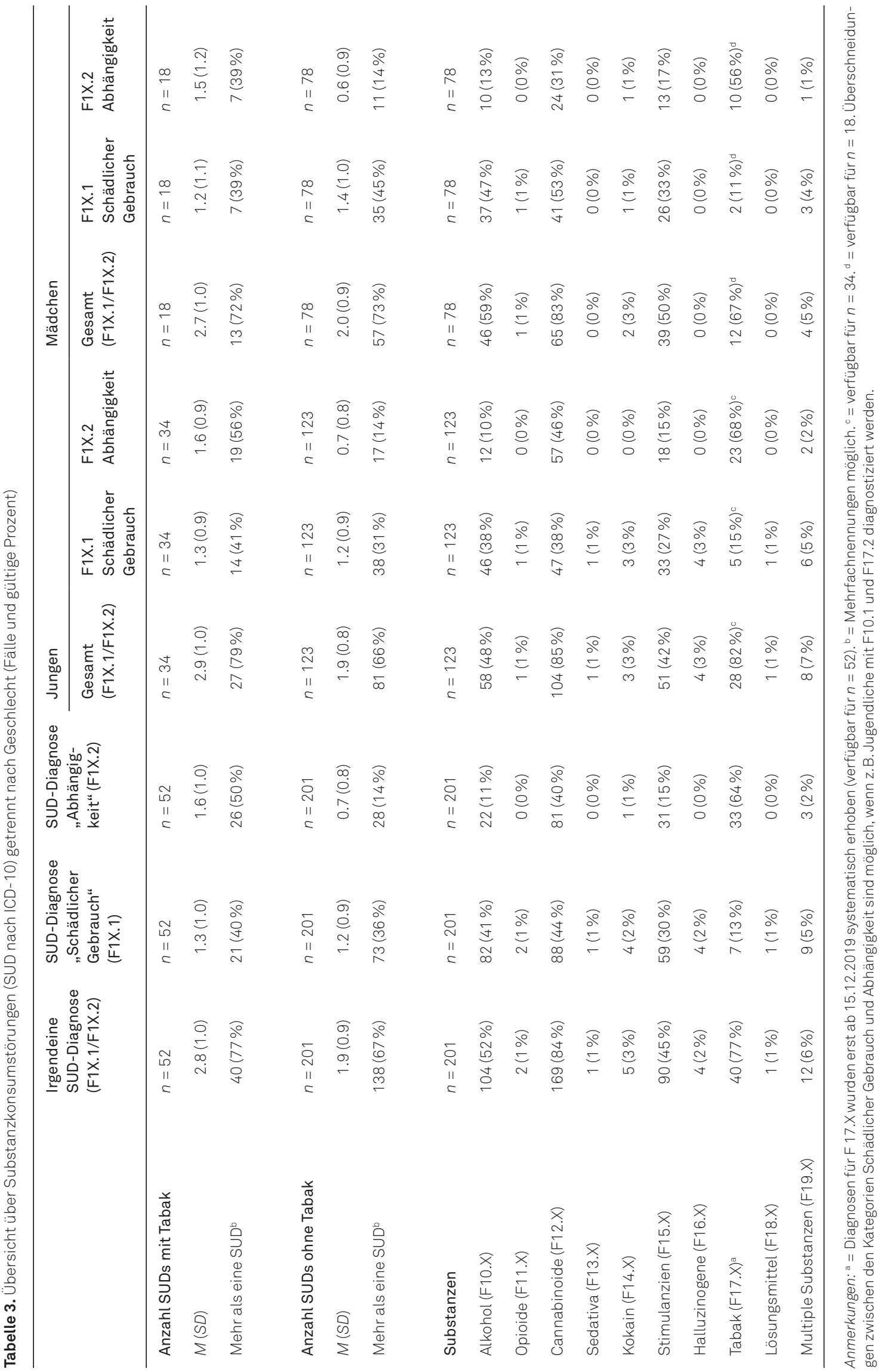




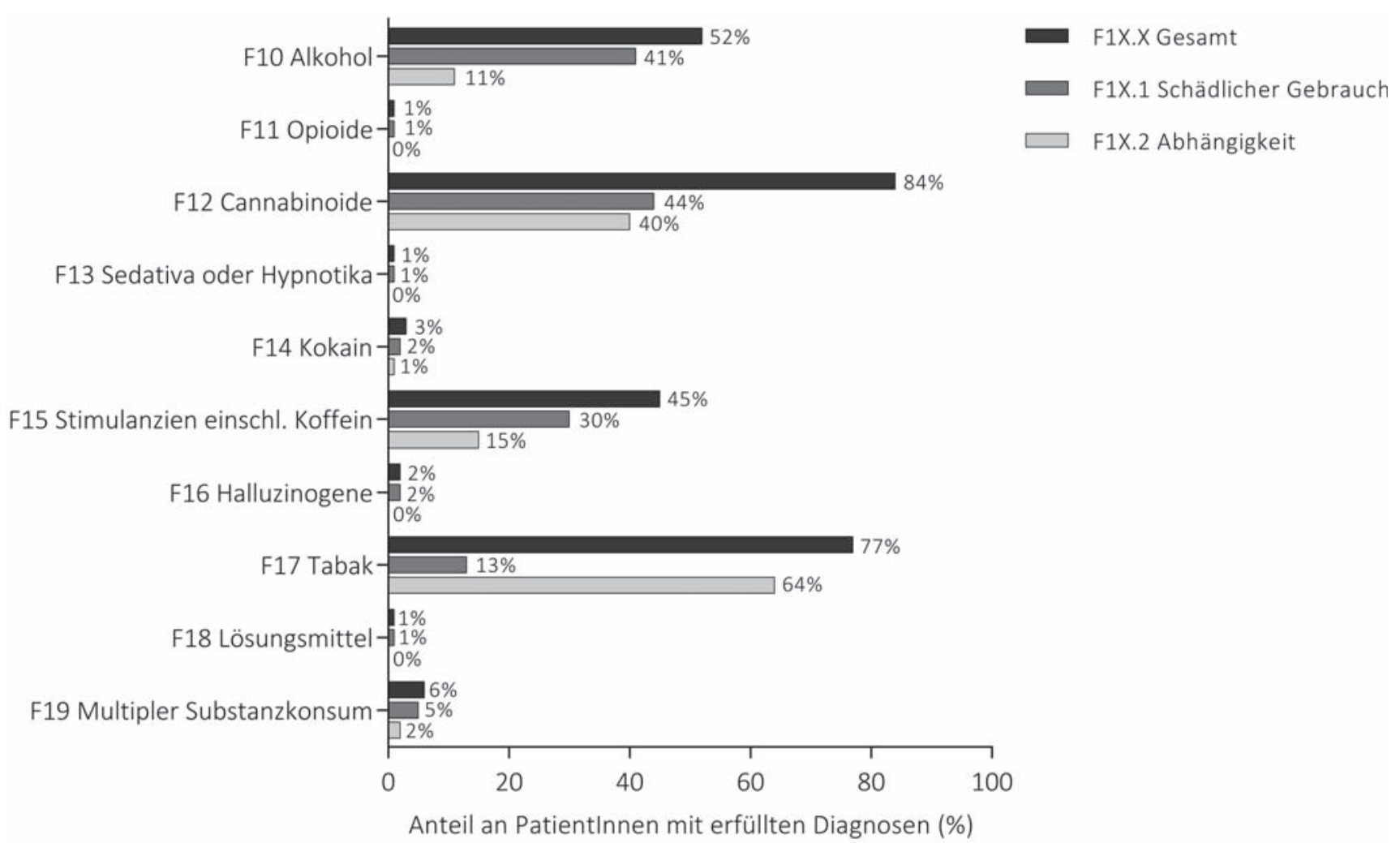

Abbildung 1. Verteilung einzelner Substanzkonsumstörungen (ICD-10) getrennt nach Schädlichem Gebrauch und Abhängigkeit für $n=196$ Patient_innen.

phetamine) den Anteil derjenigen mit Abhängigkeit (11\% für Alkohol bzw. 15\% für Amphetamine). Die Häufigkeit der SUDs war unabhängig von Geschlecht und Altersklasse (siehe Tabelle 3 und ESM 3, Tabelle).

\section{Begleitende psychische Störungen, Suizidalität und NSSV}

$72 \%$ aller Patient_innen erfüllten die Kriterien für mindestens eine begleitende nichtsubstanzbezogene psychische Störung (siehe Tabelle 4). Die am häufigsten vergebenen begleitenden Störungen waren Störungen des Sozialverhaltens (40\%), depressive Störungen (18\%), hyperkinetische Störungen (21\%) und Persönlichkeitsentwicklungsstörungen (12\%). Geschlechtsunterschiede zeigten sich hinsichtlich depressiver Störungen $(O R=7.4,95 \% \mathrm{KI}=2.4-$ 22.7, $p<.001)$ und Persönlichkeitsentwicklungsstörungen $(O R=4.1,95 \% \mathrm{KI}=1.2-13.8, p<.025)$, die jeweils bei Mädchen häufiger als bei Jungen diagnostiziert wurden. In Bezug auf begleitende nichtsubstanzbezogene psychische Störungen zeigten sich keine Unterschiede zwischen jüngeren und älteren Patient_innen (siehe ESM 4, Tabelle).

$69 \%$ der Mädchen zeigten NSSV (siehe Tabelle 4) und unterschieden sich darin deutlich von Jungen, von denen $15 \%$ NSSV zeigten $(O R=7.0,95 \% \mathrm{KI}=2.2-22.5, p=.001)$.
$26 \%$ aller Patient innen berichteten suizidale Gedanken im letzten Monat. In Bezug auf Suizidalität, NSSV oder begleitende psychische Störungen zeigten sich keine Unterschiede zwischen jüngeren und älteren Patient_innen (siehe ESM 4, Tabelle).

In einer zusätzlichen Analyse zeigte sich, dass Jugendliche, deren Eltern nicht im selben Haushalt leben, mit $41 \%$ und $O R=2.1(p=.143)$ ein tendenziell doppelt so hohes Risiko aufweisen, eine Störung des Sozialverhaltens zu zeigen, verglichen mit Jugendlichen, die mit beiden Eltern im selben Haushalt wohnen (25\%; siehe ESM 5, Tabelle). Der Zusammenhang zwischen dem Auftreten von NSSV und dem Geschlecht blieb auch unter Aufnahme der Diagnose einer Persönlichkeitsstörung bestehen (siehe ESM 6, Tabelle). Das Vorliegen einer Persönlichkeitsstörung war mit einem tendenziell 6-fachen Risiko für NSSV assoziiert $(O R=6.4, p=.057)$.

\section{Diskussion}

Die vorliegende unizentrische Studie beschreibt das Ausmaß des Substanzkonsums, Prävalenzen von Substanzkonsumstörungen sowie begleitende psychische Störungen bei Jugendlichen mit SUDs, die sich in einer 
Tabelle 4. Ergebnisse der beiden logistischen Regressionen zu Geschlechtsunterschieden bezüglich aktueller psychische Auffälligkeiten bzw. begleitender psychischer Störungena (Fälle und gültige Prozent)

\begin{tabular}{|c|c|c|c|c|c|c|c|}
\hline & Gesamt & Jungen & Mädchen & $\mathrm{B}$ & $\mathrm{p}$ & OR & {$[95 \% \mathrm{KI}]$} \\
\hline Psychische Auffälligkeiten & $n=105$ & $n=59$ & $n=46$ & & & & \\
\hline Suizidalität ${ }^{a}$ & $27(26 \%)$ & $10(17 \%)$ & $17(37 \%)$ & 1.39 & .130 & 4.0 & {$[0.7 ; 24.0]$} \\
\hline \multirow[t]{2}{*}{ Suizidversucha(1 missing) } & $24(23 \%)$ & $10(17 \%)$ & $14(31 \%)$ & -0.41 & .638 & 0.7 & {$[0.1 ; 3.6]$} \\
\hline & $n=121$ & $n=67$ & $n=54$ & & & & \\
\hline NSSV ${ }^{b}$ & $47(39 \%)$ & $10(15 \%)$ & $37(69 \%)$ & 1.95 & $.001 *$ & 7.0 & {$[2.2 ; 22.5]$} \\
\hline $\begin{array}{l}\text { Begleitende aktuelle } \\
\text { psychische Störungen }{ }^{\circ}(I C D-10)\end{array}$ & $n=201$ & $n=123$ & $n=78$ & & & & \\
\hline $\begin{array}{l}\text { Irgendeine der folgenden } \\
\text { Störungsklassen }\end{array}$ & $144(72 \%)$ & $82(67 \%)$ & $62(80 \%)$ & 0.87 & .183 & 2.4 & {$[0.7 ; 8.5]$} \\
\hline depressive Störungen & $36(18 \%)$ & $8(7 \%)$ & $28(36 \%)$ & 2.00 & $<.001^{\star}$ & 7.4 & {$[2.4 ; 22.7]$} \\
\hline Angststörungen & $6(3 \%)$ & $4(3 \%)$ & $2(3 \%)$ & -1.54 & .154 & 0.2 & {$[<0.1 ; 1.8]$} \\
\hline PTBS & $7(4 \%)$ & $1(1 \%)$ & $6(7 \%)$ & 2.23 & .086 & 9.3 & {$[0.7 ; 118.1]$} \\
\hline hyperkinetische Störungen & $42(21 \%)$ & $35(29 \%)$ & $8(10 \%)$ & -1.73 & $.001 *$ & 0.2 & {$[0.1 ; 0.5]$} \\
\hline Störung des Sozialverhaltens & $81(40 \%)$ & $55(45 \%)$ & $26(33 \%)$ & -0.73 & .196 & 0.5 & {$[0.2 ; 1.5]$} \\
\hline $\begin{array}{l}\text { Persönlichkeitsentwicklungs- } \\
\text { störungen }\end{array}$ & $25(12 \%)$ & $7(6 \%)$ & $18(23 \%)$ & 1.40 & $.025^{\star}$ & 4.1 & {$[1.2 ; 13.8]$} \\
\hline psychotische Störungen & $7(4 \%)$ & $6(5 \%)$ & $1(1 \%)$ & -2.22 & .061 & 0.1 & {$[<0.1 ; 1.1]$} \\
\hline
\end{tabular}

Anmerkungen: $\mathrm{B}=$ unstandardisierter Koeffizient, $\mathrm{KI}=$ Konfidenzintervall, NSSV = Nichtsuizidales selbstverletzendes Verhalten. OR $=$ odds ratio, PTBS = Posttraumatische Belastungsstörung. ${ }^{a}=$ Angaben aus Mini-Kid, verfügbar für insgesamt $n=105$ Proband_innen. ${ }^{b}=$ wurde im Rahmen der körperlichen Untersuchung erhoben. ${ }^{\circ}=$ begleitende Störung zu Substanzkonsumstörungen, Überschneidungen zwischen den Kategorien möglich; ${ }^{\star}=p<.025$ (Bonferroni-Korrektur, .05 geteilt durch 2, da zwei Regressionen zu Geschlechtsunterschieden berechnet wurden).

universitären Spezialambulanz für Suchterkrankungen vorstellten. Die häufigsten begleitenden Störungen waren Störung des Sozialverhaltens, hyperkinetische Störungen und depressive Störungen. Die am häufigsten konsumierten Substanzen waren Tabak und Cannabis. Die häufigsten substanzbezogenen Diagnosen waren psychische und Verhaltensstörungen durch Cannabinoide. Fast zwei Drittel aller Patient_innen zeigte mindestens eine nichtsuchtbezogene begleitende psychische Störung. Damit spiegeln die Ergebnisse nicht die Entwicklung des Konsumverhaltens der jugendlichen Allgemeinbevölkerung wider (z.B. stark reduziertes Rauchverhalten; Orth \& Merkel, 2020) und entsprechen auch nicht gänzlich der Inanspruchnahmestruktur der ambulanten Angebote in der Zielregion (häufigster Anlass mit $49 \%$ der Beratungsbedarfe aufgrund von Alkoholkonsum, 41\% aufgrund von illegalen Substanzen; Landeshauptstadt Dresden \& Gesundheitsamt, 2020), was dafür sprechen könnte, dass ein Teil von behandlungsbedürftigen Jugendlichen noch nicht vom Hilfesystem für Suchterkrankte erreicht wird.

\section{Substanzkonsum}

Die vorliegenden Ergebnisse zeigten, dass die am häufigsten konsumierten Substanzen unter allen jugendlichen Patient_innen Tabak und Cannabis waren. Die Patient_innen in der vorliegenden Stichprobe berichteten einen Konsum an nahezu jedem zweiten Tag im vergangenen Monat (13.6 Tage während der letzten 30 Tage), was etwas geringer ist als in einer Studie von Rigter et al. (2013) bei Jugendlichen, die sich in ambulante suchtspezifische Therapie in Berlin begaben und angaben, an ca. 62 der letzten 90 Tage Cannabis konsumiert zu haben, welches umgerechnet auf 30 Tage ca. 20 Konsumtagen entspricht. Das kann an regionalen Besonderheiten liegen, da Berlin im Vergleich zum Rest von Deutschland deutlich höhere Prävalenzzahlen bei Cannabiskonsum bei Jugendlichen aufweist. In Berlin gaben $16 \%$ der 15- bis 17-Jährigen an, in den letzten 12 Monaten Cannabis konsumiert zu haben (Kraus, Seitz \& Rauschert, 2020), was fast mehr als doppelt so viele Jugendliche im Vergleich zu Gesamtdeutschland (8\% der 12- bis 17-Jäh- 
rigen; Orth \& Merkel, 2020) oder Sachsen (9\%; Piontek et al., 2017) sind. Mit $70 \%$ aller Patient_innen haben in den vergangenen 12 Monaten erwartungsgemäß mehr Personen mindestens einmal Alkohol getrunken, als 12- bis 17-Jährige in der Allgemeinbevölkerung, von denen 53\% im letzten Jahr Alkohol getrunken haben (Orth \& Merkel, 2020). Die neben Cannabis am häufigsten konsumierte illegale Substanz war ähnlich wie in Studien an der Allgemeinbevölkerung MDMA mit 31\% verglichen mit 0.5\% (Orth \& Merkel, 2020). Mädchen berichteten zudem tendenziell häufiger und an mehr Tagen MDMA konsumiert zu haben als Jungen. Der höhere MDMA-Konsum bei Mädchen könnte in Zusammenhang mit einer stärkeren Belastung durch traumatische Erfahrungen stehen. Zwar sind Eigenberichten zufolge Mädchen in Deutschland insgesamt weniger häufig von Gewalterfahrungen betroffen als Jungen (Bergmann, Kliem, Krieg \& Beckmann, 2019), dennoch berichten Mädchen in Suchtkliniken häufiger Gewaltund traumatisierende Erfahrungen (Zenker et al., 2002). Da MDMA in Verbindung zu Selbstmedikationszwecken gebracht wird und als mögliche Intervention in der traumafokussierten Arbeit diskutiert wird (Thal \& Lommen, 2018), scheint dieser Zusammenhang zumindest für Personen naheliegend, die häufiger traumatischere Erlebnisse berichten und betont eine zusätzliche Beachtung in der suchtspezifischen Behandlung für adoleszente Mädchen.

\section{SUDs}

Die Häufung von Störungen durch Cannabinoide in der vorliegenden Stichprobe (85\%) entspricht in etwa dem Wert einer irischen Stichprobe (72\%) von Adoleszenten mit SUDs, die sich aufgrund von psychischen und Verhaltensstörungen durch Cannabinoide in ambulanter Behandlung befanden (Gamage, Darker \& Smyth, 2021). Auch spiegeln die Ergebnisse gleichzeitig die Daten der deutschen Suchthilfestatistik wider (Thaller et al., 2016), nach der die Inanspruchnahme ambulanter und stationärer Suchthilfen bei den bis zu 17-Jährigen zum größten Teil cannabisbezogene Behandlungsanlässe ausmachten. Psychische und Verhaltensstörungen durch Tabak lagen ebenfalls bei den meisten Patient_innen vor, waren allerdings in keinem einzigen Fall der Behandlungsanlass. Mögliche Ursachen für diese Unterversorgung umfassen stärkere psychosoziale Auswirkungen der anderen Problemsubstanzen, die Unterschätzung der langfristigen $\mathrm{Ge}$ sundheitsgefahren mit durchschnittlich 10 Jahren Lebenszeitverlust (Jha et al., 2013) und die teils mangelnde Umsetzung von Tabakkontrollmaßnahmen in deutschen Kinder- und Jugendpsychiatrien (Kuitunen-Paul et al., 2021). In der vorliegenden Stichprobe erfüllte fast jede/r Zweite die Kriterien für eine psychische und Verhaltens- störungen durch Alkohol (53\%) oder Stimulanzien (45\%), wobei die Zahl derer mit einer Abhängigkeitsdiagnose bei Stimulanzien geringfügig höher lag als bei Alkohol (15\% für Amphetamine verglichen mit 11\% für Alkohol). 77\% aller Jugendlichen wiesen eine SUD von mehr als einer Substanz auf. Diese Zahlen liegen in einem ähnlichen Bereich wie eine Studie von Reis et al. (2006), die bei Jugendlichen im Rahmen eines niedrigschwelligen Angebots für drogenkonsumierende Jugendliche und junge Erwachsene bei $81 \%$ einen polyvalenten Konsum feststellten, und zeigen, dass polyvalenter Konsum bei hilfesuchenden Jugendlichen eher die Regel als die Ausnahme ist. Wie in der Studie von Reis et al. (2006) beschrieben lassen sich polyvalente Konsummuster mit speziellen begleitenden Störungen assoziieren, insbesondere mit hyperkinetischen Störungen, Störungen des Sozialverhaltens und der Kombinierten Störung des Sozialverhaltens und der Emotionen. Künftige Untersuchungen sollten daher bei der Untersuchung begleitender psychischer Störungen polyvalenten Konsum sowie das Vorliegen von mehr als einer SUD berücksichtigen.

\section{Begleitende psychische Störungen}

Knapp zwei Drittel (65\%) der untersuchten Jugendlichen erfüllte die Kriterien für mindestens eine weitere nichtsubstanzbezogene Diagnose. Diese Zahlen liegen deutlich höher als bei Patient_innen mit SUD aus nichtsuchtspezifischen Krankenhäusern, bei denen Prävalenzzahlen in psychischen Notaufnahmen zwischen 17 und $34 \%$ variieren (de Veld, van Hoof, Wolberink \& van der Lely, 2021; Haw \& Hawton, 2011; Martín-Santos et al., 2006). Dies liegt wahrscheinlich daran, dass bei Jugendlichen ein problematischer Substanzkonsum von den Sorgeberechtigten häufig nicht erkannt oder als nicht behandlungswürdig empfunden wird, was vor allem zutrifft, wenn keine zusätzlichen Verhaltensauffälligkeiten vorliegen. Demnach würden viele Jugendliche mit SUD ohne begleitende psychische Auffälligkeiten in der Spezialambulanz erst gar nicht vorgestellt werden, was die hohen Prävalenzzahlen begleitender psychischer Störungen erklären würde. Dafür spricht auch, dass Störungen des Sozialverhaltens, die durch ihr dissoziales Verhalten besonders auffallen, die häufigste begleitende Diagnose mit einer Prävalenz von $38 \%$ unter allen Patient_innen war. Die Prävalenzzahlen für begleitende Störungen des Sozialverhaltens und hyperkinetische Störungen liegen in der vorliegenden Studie deutlich unter denen anderer Studien, die Prävalenzzahlen von $74 \%$ für Störungen des Sozialverhaltens und $77 \%$ für hyperkinetische Störungen in ambulanten Einrichtungen für SUDs von Cannabis finden (Diamond et al., 2006). Der Grund hierfür könnte ebenfalls ein Selektionseffekt 
sein. Cannabis wird verglichen mit anderen illegalen Substanzen (Sarkar et al., 2014) und über die letzten Jahre von Jugendlichen zunehmend als weniger schädlich wahrgenommen, wie eine Studie aus Norwegen zeigte (Burdzovic Andreas, 2019). Demnach würden Jugendliche ohne externalisierende Verhaltensauffälligkeiten mit geringerer Wahrscheinlichkeit zur Behandlung vorgestellt werden. Die Prävalenz für eine begleitende Depression lag in der Studie von Diamond et al. (2006) mit 29 \% etwas höher als in der vorliegenden Studie. Dies kann daran liegen, dass hoher Cannabiskonsum mit depressiver Symptomatik in Verbindung gebracht wird bzw. zu depressiven Symptomen führen kann (Feingold \& Weinstein, 2021) und es in dieser Studie zu o.g. Selektionseffekt kommt. Depressive Störungen wurden in der vorliegenden Studie häufiger bei Mädchen diagnostiziert. Die Ergebnisse stehen in Kontrast zu Befunden einer Studie aus Spanien, die weder bei High-School-Schüler_Innen noch bei Jugendlichen, die die Kriterien für eine Alkohol- und Cannabiskonsumstörung erfüllten, Unterschiede zwischen Jungen und Mädchen im Risiko, an einer Depression zu leiden, fand (Fernández-Artamendi, Martínez-Loredo \& LópezNúñez, 2021). Dies könnte daran liegen, dass die Studie von Fernández-Artamendi et al. (2021) durch die Verwendung von Fragebögen Depressivität besser erfassen konnte. Depressionen sind bei Männern, zum Teil bedingt durch erhöhte Selbststigmatisierung (Latalova et al., 2014) und Unterschiede in der Ausprägung, häufig unterdiagnostiziert. Dies ist vor allem der Fall, wenn der Fokus der Behandlung auf anderen Verhaltensweisen, wie in der vorliegenden Studie der Suchtproblematik, liegt und das könnte dazu führen, dass Depressionen bei männlichen Jugendlichen öfter übersehen werden.

Im Gegensatz zu einigen Vorbefunden, die zeigen, dass Jungen häufiger an externalisierenden Störungen (Störungen des Sozialverhaltens und hyperkinetische Störungen) leiden (Mendez et al., 2021), finden sich in der vorliegenden Stichprobe von ambulant behandelten substanzkonsumierenden Jugendlichen keine diesbezüglichen geschlechtsspezifischen Unterschiede. Das könnte vor allem daran liegen, dass besonders Mädchen, die an SUDs leiden, ein erhöhtes Risiko für externalisierendes Verhalten aufweisen (Korsgaard, Torgersen, Wentzel-Larsen \& Ulberg, 2016) und Studien an SUD-Patient_innen überproportional viele Mädchen mit Störungen des Sozialverhaltens vorfinden. Das gehäufte Vorkommen von Störungen des Sozialverhaltens kann erklärt werden durch den größeren Anteil an männlichen Jugendlichen bzw. den hohen Anteil an Jugendlichen, deren Eltern getrennt leben. Beide Merkmale treten in unserer Stichprobe häufiger auf als in der Allgemeinbevölkerung des Studienorts (Landeshauptstadt Dresden, Kommunale Statistikstelle, 2019) und sind assoziiert mit dem Auftreten des Störungsbildes (Berkout, Young \&
Gross, 2011; Brüggemann, 2016). Dies wird durch die Tatsache unterstrichen, dass Jugendliche in unserer Stichprobe, die nicht mehr bei beiden Eltern im selben Haushalt wohnten, ein tendenziell doppelt so hohes Risiko aufwiesen, eine Störung des Sozialverhaltens zu zeigen. Diese Ergebnisse unterstreichen die Wichtigkeit sozioökonomischer Einflüsse auf begleitende psychische Erkrankungen. Daher sollten künftige Untersuchungen sozioökonomische Aspekte wie Migration, Trennung, Schulbesuch etc. als mögliche Risikofaktoren für die Entstehung von SUDs und begleitenden psychischen Erkrankungen miteinbeziehen.

Die vorliegenden Ergebnisse weisen darauf hin, dass ambulant vorstellige, chronisch substanzkonsumierende, jugendliche Mädchen im Vergleich zu Jungen ein erhöhtes Risiko für depressive Störungen, Persönlichkeitsentwicklungsstörungen und wiederholtes NSSV aufweisen. Dieser geschlechtsspezifische Unterschied bei NSSV wird auch von epidemiologischen Studien berichtet (Bresin \& Schoenleber, 2015; Moran et al., 2015) und scheint ein geschlechterspezifisches und kein suchtspezifisches Phänomen zu sein. Eine systematische Übersichtsarbeit von Miranda-Mendizabal et al. (2019) zeigte, dass weibliche Patient_innen mit SUD im Vergleich zu Patient_innen ohne SUD ein 4.4-fach (95\% KI = 2.5-7.8) erhöhtes Risiko für NSSV ausweisen. Männliche Patienten mit SUD liegen mit einem 3.1-fach (95\% KI = 2.0-4.8) erhöhtem Risiko etwas niedriger, aber in einem vergleichbaren Bereich. In nichtklinischen Populationen an Schulkindern in Deutschland reichen die Prävalenzraten von 7\% (Jungen) bis 17\% (Mädchen; Brunner et al., 2014). Die Prävalenz für NSSV in der vorliegenden Stichprobe lag mit $15 \%$ für Jungen in einem doppelt so hohen und für Mädchen mit $69 \%$ in einem vierfach so hohen Bereich wie in der Arbeit von Brunner et al. (2014). Die häufig beobachteten geschlechtsspezifischen Unterschiede zu NSSV (Plener et al., 2015) spiegeln sich auch in unserer Stichprobe wider und unterstreichen das besondere Risiko für NSSV bei jugendlichen Mädchen. Diese Unterschiede könnten auch in Verbindung zu Persönlichkeitsentwicklungsstörungen wie der Borderline-Persönlichkeitsstörung stehen. Diese treten sowohl bei SUDs als auch NSSV (Ghinea et al., 2020) und bei Mädchen häufiger auf (Chabrol, Montovany, Chouicha, Callahan \& Mullet, 2001). Es zeigte sich in einer zusätzlichen Analyse, dass das Vorliegen einer Persönlichkeitsstörung zwar mit einem tendenziell sechsfach erhöhten Risiko für NSSV verbunden war $(\mathrm{OR}=6.4, p=.057)$, dennoch wiesen Mädchen auch unter Hinzunahme der Variable Persönlichkeitsstörungen ein signifikant erhöhtes Risiko für NSSV auf, was darauf hindeutet, dass Mädchen unabhängig von Persönlichkeitsstörungen ein erhöhtes Risiko für NSSV aufweisen. Für die Behandlung einer Borderline-Persönlichkeitsstörung bei Jugendlichen wird nach den S2-Leitlinien die 
Dialektisch-Behaviorale Therapie für Adoleszente empfohlen (Renneberg et al., 2010). Stationäre sowie ambulante Einrichtungen sind jedoch in Deutschland nur sehr eingeschränkt verfügbar, besonders solche die sich auf die Kombination Suchterkrankung/Borderline-Persönlichkeitsstörung spezialisiert haben.

Trotz dieser hohen Prävalenzzahlen begleitender psychischer Störungen existieren, beispielsweise bei Jugendlichen mit Methamphetaminkonsumstörungen, nur wenige solcher Therapieprogramme (Kuitunen-Paul et al., 2021). Die S3-Leitlinien für die Behandlung von Alkoholproblemen bzw. Methamphetaminkonsumstörungen empfehlen bei allen begleitenden psychischen Störungen eine integrierte Behandlung (Deutsche Gesellschaft für Psychiatrie und Psychotherapie, Psychosomatik und Nervenheilkunde e.V. [DGPPN], 2020). Sollte eine integrierte Behandlung nicht möglich sein, ist eine enge Abstimmung zwischen verschiedenen Behandler_innen bei paralleler oder sequenzieller Behandlung von SUDs und begleitenden psychischen Störungen besonders wichtig. Beispielsweise kann eine Entwöhnungsbehandlung - kurzfristig gesehen - eine Verschlechterung einer der SUD vorausgehenden begleitenden Depression verursachen und muss dementsprechend in der Folgebehandlung berücksichtigt werden (Thase et al., 2001). Auch kann es vorkommen, dass begleitende Störungen erst nach einer erfolgreichen Entwöhnung auftreten und erkannt werden, weshalb eine diagnostische Einordnung von Depressionen beispielsweise bei Alkoholkonsumstörungen erst nach 3 bis 4 Wochen empfohlen wird (Haber, Litzeris, Proude \& Lopatko, 2009). In Anbetracht der langen Wartezeiten auf eine ambulante Behandlung bei Psychotherapeut_innen ist es bereits zum Zeitpunkt der ersten Vorstellung wichtig, den Fokus auch auf begleitende Störungen zu legen, um langfristig die Therapie planen zu können. Dabei ist es hilfreich, bei besonders vulnerablen Gruppen begleitende Störungen zu identifizierten. An dieser Stelle können sogenannte Stepped-Care-Modelle in der Behandlung zukunftsweisend sein (Rumpf et al., 2016; Bühringer \& Rumpf, 2018), mithilfe derer Patient_innen in Abhängigkeit der Schwere der Störung und/oder begleitenden Störungen auf der jeweiligen Interventionsstufe behandelt werden. Um hierfür entscheiden zu können, welche Hilfen gerade akut eingeleitet und welche längerfristig geplant werden sollen, ist es wichtig, frühzeitig begleitende Störungen zu identifizieren.

\section{Limitationen}

Die vorliegenden Daten beziehen sich auf Jugendliche die sich wegen Verdacht auf das Vorliegen einer Substanzkonsumstörung in unserer Ambulanz vorstellten und sind somit nicht auf die deutsche Gesamtpopulation an jugendlichen Konsument_innen übertragbar. Die Jugendlichen stammen mit $84 \%$ verglichen mit der Gesamtpopulation im Studiengebiet (22\%; Landeshauptstadt Dresden, Kommunale Statistikstelle, 2019) überproportional häufig aus Familien, in denen beide Elternteile nicht mehr in einem gemeinsamen Haushalt wohnen. Das hohe Vorkommen an Jugendlichen, die nicht mehr mit beiden Eltern im selben Haushalt wohnen, ähnelt Studien, die ambulante Hilfen bei Verdacht auf Substanzkonsumstörungen im deutschsprachigen Raum anbieten (Reis et al., 2009). Dennoch weist unsere Stichprobe mit $10 \%$ einen ähnlichen Anteil an Jugendlichen wie die Gesamtbevölkerung des Untersuchungsgebiets auf, die die Schule ohne Abschluss verlassen (8\%; Landeshauptstadt Dresden, Kommunale Statistikstelle, 2019), und stellen zumindest in diesem Punkt ein repräsentatives Bild dar. Die vorliegenden Daten beziehen sich außerdem lediglich auf Jugendliche im Alter zwischen 12 und 19 Jahren, somit kann keine Aussage über jüngere Kinder oder junge Erwachsene getroffen werden. Da die Prävalenzzahlen für Sachsen beim Alkohol- und Methamphetaminkonsum in den vergangenen Jahren höher waren als im Rest von Deutschland (Pfeiffer-Gerschel et al., 2019; Sächsisches Staatsministerium für Soziales und Gesellschaftlichen Zusammenhalt, 2019), ist die Generalisierbarkeit bzgl. Gesamtdeutschland bei diesen Substanzen eingeschränkt. Abschließend muss beachtet werden, dass die Zusammenhänge zwischen Substanzkonsum und begleitenden Störungen lediglich im Querschnittsansatz untersucht wurden und keine Kausalaussagen in Bezug auf unabhängige begleitende Störungen und substanzinduzierte begleitende Störungen getroffen werden können, welche in Zukunft in Längsschnittstudien untersucht werden sollten.

\section{Fazit für die klinische Praxis}

Ambulante Behandlungsangebote für Jugendliche mit SUDs sollten ihr Angebot vor allem in Bezug auf die Behandlung begleitender Störungen des Sozialverhaltens, depressiven Störungen und hyperkinetische Störungen erweitern. Leider gibt es in Deutschland trotz der steigenden Inanspruchnahme (Sächsische Landesstelle gegen die Suchtgefahren, 2021) in den letzten Jahren wenig ambulante suchtspezifische Einrichtungen. Im Gegenteil müssen Betroffene in nichtsuchtspezifischen Einrichtungen häufig zusätzliche Hürden nehmen, bis ein Therapieplatz wahrgenommen werden kann. Beispielsweise müssen Patient_innen vor einer Behandlung einer begleitenden Depression abstinent sein, was in vielen Fällen ohne thera- 
peutische Unterstützung nicht geleistet werden kann. Hierfür empfehlen sich aufklärende und entstigmatisierende Inhalte für Pflegende und Behandelnde (KuitunenPaul et al., 2021) ebenso wie die Schaffung integrativer Behandlungsangebote (z.B. Golub et al., 2021), die Abstinenz als Ziel statt als Voraussetzung ansehen (Bühringer \& Rumpf, 2018; Rumpf et al., 2017).

\section{Elektronisches Supplement}

Das elektronische Supplement (ESM) ist mit der Onlineversion dieses Artikels verfügbar unter https://doi.org/10.1024/1422-4917/a000846.

ESM 1. Durchschnittliche Konsumtage während des letzten Monats

ESM 2. Mittleres Alter bei Erstgebrauch der Substanz

ESM 3. Übersicht über Substanzkonsumstörungen

ESM 4. Ergebnisse der beiden logistischen Regressionen zu Geschlechtsunterschieden bzgl. begleitender psychischer Störungen

ESM 5. Ergebnisse der logistischen Regression zu Altersunterschieden bzgl. begleitender psychischer Störungen

ESM 6. Ergebnisse der beiden logistischen Regressionen zu Geschlechtsunterschieden bzgl. begleitender psychischer Störungen (inklusive Persönlichkeitsstörungen)

\section{Literatur}

Armstrong, T.D. \& Costello, E.J. (2002). Community studies on adolescent substance use, abuse, or dependence and psychiatric comorbidity. Journal of Consulting and Clinical Psychology, 70, 1224-1239. https://doi.org/10.1037/0022-006X.70.6.1224

Beesdo-Baum, K., Knappe, S., Asselmann, E., Zimmermann, P., Brückl, T., Höfler, M. et al. (2015). The „Early Developmental Stages of Psychopathology (EDSP) study“: A 20-year review of methods and findings. Social Psychiatry and Psychiatric Epidemiology, 50, 851-866. https://doi.org/10.1007/s00127-015-1062-x

Behrendt, S., Wittchen, H.-U., Höfler, M., Lieb, R., Low, N.C.P., Rehm, J. et al. (2008). Risk and speed of transitions to first alcohol dependence symptoms in adolescents: A 10-year longitudinal community study in Germany. Addiction, 103, 1638-1647. https://doi.org/10.1111/j.1360-0443.2008.02324.x

Bergmann, M. C., Kliem, S., Krieg, Y. \& Beckmann, L. (2019). Jugendliche in Niedersachsen. Ergebnisse des Niedersachsensurveys 2017 (Forschungsbericht Nr. 144). Hannover: Kriminologisches Forschungsinstitut Niedersachsen e.V. Verfügbar unter https:// kfn.de/wp-content/uploads/Forschungsberichte/FB_144.pdf

Berkout, O., Young, J. \& Gross, A. (2011). Mean girls and bad boys: Recent research on gender differences in conduct disorderScienceDirect. Aggression and Violent Behavior, 16, 503-511.

Bresin, K. \& Schoenleber, M. (2015). Gender differences in the prevalence of nonsuicidal self-injury: A meta-analysis. Clinical Psychology Review, 38, 55-64. https://doi.org/10.1016/j.cpr.2015.02.009
Brüggemann, K. (2016). Symptombelastung und die Rolle von Freundschaften bei Kindern in einer Erziehungsberatungsstelle aus Elternsicht. Praxis der Kinderpsychologie und Kinderpsychiatrie, 65,127-142. https://doi.org/10.13109/prkk.2016.65.2.127

Brunner, R., Kaess, M., Parzer, P., Fischer, G., Carli, V., Hoven, C. W. et al. (2014). Life-time prevalence and psychosocial correlates of adolescent direct self-injurious behavior: A comparative study of findings in 11 European countries. Journal of Child Psychology and Psychiatry, and Allied Disciplines, 55, 337-348. https:// doi.org/10.1111/jcpp.12166

Bühringer, G. \& Rumpf, H.-J. (2018). Zukunft der Suchtkrankenversorgung: Plädoyer für einen Paradigma-Wechsel. Future of addiction treatment: Plea for a paradigmatic change. Sucht, 64, 125-128. https://doi.org/10.1024/0939-5911/a000536

Bukstein, O.G., Glancy, L.J. \& Kaminer, Y. (1992). Patterns of affective comorbidity in a clinical population of dually diagnosed adolescent substance abusers. Journal of the American Academy of Child \& Adolescent Psychiatry, 31, 1041-1045. https://doi. org/10.1097/00004583-199211000-00007

Burdzovic Andreas, J. (2019). Perceived harmfulness of various alcohol- and cannabis use modes: Secular trends, differences, and associations with actual substance use behaviors among Norwegian adolescents, 2007-2015. Drug and Alcohol Dependence, 197, 280-287. https://doi.org/10.1016/j.drugalcdep.201 9.02 .003

Chabrol, H., Montovany, A., Chouicha, K., Callahan, S. \& Mullet, E. (2001). Frequency of borderline personality disorder in a sample of French high school students. Canadian Journal of Psychiatry, 46, 847-849. https://doi.org/10.1177/070674370104600909

Chan, Y.-F., Dennis, M.L. \& Funk, R.R. (2008). Prevalence and comorbidity of major internalizing and externalizing problems among adolescents and adults presenting to substance abuse treatment. Journal of Substance Abuse Treatment, 34, 14-24. https://doi.org/10.1016/j.jsat.2006.12.031

Chen, L.-Y., Strain, E.C., Crum, R. M. \& Mojtabai, R. (2013). Gender differences in substance abuse treatment and barriers to care among persons with substance use disorders with and without comorbid major depression. Journal of Addiction Medicine, 7 , 325-334. https://doi.org/10.1097/ADM.0b013e31829b7afe

Choi, H.J., Lu, Y., Schulte, M. \& Temple, J.R. (2018). Adolescent substance use: Latent class and transition analysis. Addictive Behaviors, 77, 160-165. https://doi.org/10.1016/j.addbeh.2017.0 9.022

Cohen, J. (2009). Statistical power analysis for the behavioral sciences (2nd ed., reprint). Hillsdale, NY: Lawrence Erlbaum Associates.

de Veld, L., van Hoof, J.J., Wolberink, I. M. \& van der Lely, N. (2021). The co-occurrence of mental disorders among Dutch adolescents admitted for acute alcohol intoxication. European Journal of Pediatrics, 180, 937-947. https://doi.org/10.1007/s0043 1-020-03823-0

Diamond, G., Panichelli-Mindel, S. M., Shera, D., Dennis, M., Tims, F. \& Ungemack, J. (2006). Psychiatric syndromes in adolescents with marijuana abuse and dependency in outpatient treatment. Journal of Child \& Adolescent Substance Abuse, 15(4), 37-54. https://doi.org/10.1300/J029v15n04_02

European Monitoring Centre for Drugs and Drug Addiction. (2015). Comorbidity of substance use and mental disorders in Europe. Luxembourg: Publications Office of the European Union. Verfügbar unter https://www.emcdda.europa.eu/system/files/publications/1988/TDXD15019ENN.pdf

Feingold, D. \& Weinstein, A. (2021). Cannabis and depression. In E. Murillo-Rodriguez, S.R. Pandi-Perumal \& J.M. Monti (Eds.), Cannabinoids and Neuropsychiatric Disorders (pp. 67-80). Cham: Springer International Publishing. https://doi.org/10.10 07/978-3-030-57369-0_5 
Fernández-Artamendi, S., Martínez-Loredo, V. \& López-Núñez, C. (2021). Sex differences in comorbidity between substance use and mental health in adolescents: Two sides of the same coin. Psicothema, 33, 36-43. https://doi.org/10.7334/psicothema20 20.297

Gamage, N.M., Darker, C. \& Smyth, B.P. (2021). Improvement in psychological wellbeing among adolescents with a substance use disorder attending an outpatient treatment programme. Irish Journal of Psychological Medicine, 1-8. https://doi.org/10. 1017/ipm.2021.6

Golub, Y., Basedow, L. A., Meiron-Zwipp, J., Kuitunen-Paul, S. \& Roessner, V. (2021). DELTA - Dresdner Multimodale Therapie für Jugendliche mit chronischem Suchtmittelkonsum. Göttingen: Hogrefe.

Gomes de Matos, E., Atzendorf, J., Kraus, L. \& Piontek, D. (2016). Substanzkonsum in der Allgemeinbevölkerung in Deutschland: Ergebnisse des Epidemiologischen Suchtsurveys 2015. Sucht, 62, 271-281. https://doi.org/10.1024/0939-5911/a000445

Gomes de Matos, E., Hannemann, T.-V., Atzendorf, J., Kraus, L. \& Piontek, D. (2018). The consumption of new psychoactive substances and methamphetamine. Analysis of data from 6 German federal states. Deutsches Ärzteblatt International, 115, 49-55. https://doi.org/10.3238/arztebl.2018.0049

Grella, C.E., Hser, Y.-I., Joshi, V. \& Rounds-Bryant, J. (2001). Drug treatment outcomes for adolescents with comorbid mental and substance use disorders. Journal of Nervous and Mental Disease, 189, 384-392. https://doi.org/10.1097/00005053-20010 6000-00006

Grella, C.E., Joshi, V. \& Hser, Y.-I. (2004). Effects of comorbidity on treatment processes and outcomes among adolescents in drug treatment programs. Journal of Child and Adolescent Substance Abuse, 13(4), 13-31. https://doi.org/10.1300/J029v13 n04_02

Haber, P., Litzeris, N., Proude, E. \& Lopatko, O. (2009). Guidelines for the treatment of alcohol problems. Canberra: Australian Government Department of Health and Ageing. Available from https://www.health.gov.au/sites/default/files/guidelines-forthe-treatment-of-alcohol-problems_0.pdf

Harris, K.M. \& Edlund, M.J. (2005). Use of mental health care and substance abuse treatment among adults with co-occurring disorders. Psychiatric Services, 56, 954-959. https://doi. org/10.1176/appi.ps.56.8.954

Haw, C. M. \& Hawton, K. (2011). Problem drug use, drug misuse and deliberate self-harm: Trends and patient characteristics, with a focus on young people, Oxford, 1993-2006. Social Psychiatry and Psychiatric Epidemiology, 46, 85-93. https://doi.org/10. 1007/s00127-009-0170-x

Holtmann, M., Buchmann, A.F., Esser, G., Schmidt, M.H., Banaschewski, T. \& Laucht, M. (2011). The Child Behavior ChecklistDysregulation Profile predicts substance use, suicidality, and functional impairment: A longitudinal analysis. Journal of Child Psychology and Psychiatry, 52, 139-147. https://doi.org/10.11 11/j.1469-7610.2010.02309.x

Hser, Y.-I., Grella, C.E., Collins, C. \& Teruya, C. (2003). Drug-use initiation and conduct disorder among adolescents in drug treatment. Journal of Adolescence, 26, 331-345. https://doi. org/10.1016/S0140-1971(03)00012-5

Jha, P., Ramasundarahettige, C., Landsman, V., Rostron, B., Thun, M., Anderson, R. N. et al. (2013). 21st-century hazards of smoking and benefits of cessation in the United States. New England Journal of Medicine, 368, 341-350. https://doi.org/10.1056/ NEJMsa1211128

Kaminer, Y. (1996). Adolescent substance abuse and suicidal behavior. Child and Adolescent Psychiatric Clinics of North America, 5, 59-71. https://doi.org/10.1016/S1056-4993(18)3 0385-7
Kipping, R.R., Campbell, R.M., MacArthur, G.J., Gunnell, D.J. \& Hickman, M. (2012). Multiple risk behaviour in adolescence. Journal of Public Health, 34(suppl_1), i1-i2. https://doi.org/10.1 093/pubmed/fdr122

Korsgaard, H.O., Torgersen, S., Wentzel-Larsen, T. \& Ulberg, R. (2016). Substance abuse and personality disorder comorbidity in adolescent outpatients: Are girls more severely ill than boys? Child and Adolescent Psychiatry and Mental Health, 10, 8. https://doi.org/10.1186/s13034-016-0096-5

Kraus, L., Seitz, N.-N. \& Rauschert, C. (2020). Epidemiologischer Suchtsurvey Berlin 2018. Ergänzende Ergebnisse zu illegalen Drogen und substanzbezogenen Störungen. München: IFT Institut für Therapieforschung. Verfügbar unter https://www.esasurvey.de/fileadmin/user_upload/Literatur/Berichte/ESA_20 18_Berlin.pdf

Kuitunen-Paul, S., Kilian, C., Binder, A., Luderer, M., Reichl, D. \& Speerforck, S. (2021). Alkohol - wie abhängig sind Hilfe und Stigmatisierung voneinander? In akzept e.V. (Hrsg.), 8. Alternativer Drogen-und Suchtbericht (S. 20-27). Lengerich: Pabst Science.

Kuitunen-Paul, S., Roessner, V., Basedow, L.A. \& Golub, Y. (2021). Beyond the tip of the iceberg: A narrative review to identify research gaps on comorbid psychiatric disorders in adolescents with methamphetamine use disorder or chronic methamphetamine use. Substance Abuse, 42, 13-32. https://doi.org/10.1080/ 08897077.2020 .1806183

Kuitunen-Paul, S., Teichmann, M., Mühlig, S., Lochbuehler, K. \& Roessner, V. (eingereicht). Umsetzung von Tabakkontrollmaßnahmen in Einrichtungen der Kinder- und Jugendpsychiatrie: Eine Online-Befragung von leitenden Mitarbeitenden.

Landeshauptstadt Dresden, Gesundheitsamt. (Hrsg.). (2020). Dresdner Suchtbericht 2020. Verfügbar unter https://www. dresden.de/media/pdf/gesundheit/SPDi/sucht/Suchtbericht_ 2020.pdf.

Landeshauptstadt Dresden, Kommunale Statistikstelle. (2019). Statistische Mitteilungen. Bevölkerung und Haushalte 2019. Verfügbar unter https://www.dresden.de/media/pdf/online shop/statistikstelle/Bevoelkerung_und_Haushalte_2019.pdf

Latalova, K., Kamaradova, D. \& Prasko, J. (2014). Perspectives on perceived stigma and self-stigma in adult male patients with depression. Neuropsychiatric Disease and Treatment, 10,13991405. https://doi.org/10.2147/NDT.S54081

Lieb, R., Schuster, P., Pfister, H., Fuetsch, M., Höfler, M., Isensee, B. et al. (2000). Epidemiologie des Konsums, Mißbrauchs und der Abhängigkeit von legalen und illegalen Drogen bei Jugendlichen und jungen Erwachsenen: Die prospektiv-longitudinale Verlaufsstudie EDSP. Sucht, 46, 18-31. https://doi.org/10.1024/ suc. 2000.46.1.18

Loeber, S., Dinter, C. \& Mann, K. (2011). Psychotherapie von Patienten mit Depression und Abhängigkeitserkrankung. Sucht, 57 , 373-381. https://doi.org/10.1024/0939-5911.a000140

Martín-Santos, R., Fonseca, F., Domingo-Salvany, A., Ginés, J.M., Ímaz, M. L., Navinés, R. et al. (2006). Dual diagnosis in the psychiatric emergency room in Spain. European Journal of Psychiatry, 20,147-156.

Mendez, B., Bozzay, M. \& Verona, E. (2021). Internalizing and externalizing symptoms and aggression and violence in men and women. Aggressive Behavior, 47, 439-452. https://doi.org/10.10 02/ab.21962

Miranda-Mendizabal, A., Castellví, P., Parés-Badell, O., Alayo, I., Almenara, J., Alonso, I. et al. (2019). Gender differences in suicidal behavior in adolescents and young adults: Systematic review and meta-analysis of longitudinal studies. International Journal of Public Health, 64, 265-283. https://doi.org/10.1007/ s00038-018-1196-1

Mokdad, A. H., Forouzanfar, M. H., Daoud, F., Mokdad, A. A., El Bcheraoui, C., Moradi-Lakeh, M. et al. (2016). Global burden of disea- 
ses, injuries, and risk factors for young people's health during 1990-2013: A systematic analysis for the Global Burden of Disease Study 2013. Lancet, 387, 2383-2401. https://doi. org/10.1016/S0140-6736(16)00648-6

Moran, P., Coffey, C., Romaniuk, H., Degenhardt, L., Borschmann, R. \& Patton, G.C. (2015). Substance use in adulthood following adolescent self-harm: A population-based cohort study. Acta Psychiatrica Scandinavica, 131, 61-68. https://doi.org/10.1111/ acps.12306

Orth, B. \& Merkel, C. (2019). Der Alkoholkonsum Jugendlicher und junger Erwachsener in Deutschland. Ergebnisse des Alkoholsurveys 2018 und Trends. BZgA-Forschungsbericht. https://doi. org/10.17623/BZGA:225-ALKSY18-ALK-DE-1.0

Orth, B. \& Merkel, C. (2020). Die Drogenaffinität Jugendlicher in der Bundesrepublik Deutschland 2019. Rauchen, Alkoholkonsum und Konsum illegaler Drogen: Aktuelle Verbreitung und Trends. BZgA-Forschungsbericht. https://doi.org/10.17623/BZGA:225DAS19-DE-1.0

Pfeiffer-Gerschel, T., Schneider, F., Dammer, E., Braun, B. \& Kraus, L. (2019). Methamphetaminkonsum in Deutschland: Verbreitung und Problemlage. Sucht, 65, 241-249. https://doi. org/10.1024/0939-5911/a000617

Piontek, D., Gomes de Matos, E., Atzendorf, J. \& Kraus, L. (2017). Substanzkonsum und Hinweise auf klinisch relevanten Konsum in Bayern, Hamburg, Hessen, Nordrhein-Westfalen, Sachsen und Thüringen. Ergebnisse des Epidemiologischen Suchtsurvey 2015 (IFT-Berichte Bd. 189). München: IFT Institut für Therapieforschung. Verfügbar unter https://www.esa-survey. de/fileadmin/user_upload/esa_laenderberichte/Bd_189_ ESA_2015.pdf

Plener, P.L., Schumacher, T.S., Munz, L.M. \& Groschwitz, R.C. (2015). The longitudinal course of non-suicidal self-injury and deliberate self-harm: A systematic review of the literature. Borderline Personality Disorder and Emotion Dysregulation, 2, 2. https://doi.org/10.1186/s40479-014-0024-3

Reis, O., Fegert, J. M. \& Häßler, F. (2006). Muster polyvalenten Drogengebrauchs bei niedrigschwellig betreuten Jugendlichen. Sucht, 52, 305-316. https://doi.org/10.1024/2006.05.04

Reis, O., Pape, M. \& Häßler, F. (2009). Ergebnisse eines Projektes zur kombinierten Prävention jugendlichen Rauschtrinkens. Sucht, 55, 347-356. http://doi.org/10.1024/2009.06.04

Renneberg, B., Schmitz, B., Doering, S., Herpertz, S. \& Bohus, M. (2010). Behandlungsleitlinie Persönlichkeitsstörungen. Psychotherapeut, 55, 339-354. https://doi.org/10.1007/s00278-01 0-0748-5

Rigter, H., Henderson, C.E., Pelc, I., Tossmann, P., Phan, O., Hendriks, V. et al. (2013). Multidimensional family therapy lowers the rate of cannabis dependence in adolescents: A randomised controlled trial in Western European outpatient settings. Drug and Alcohol Dependence, 130, 85-93. https://doi.org/10.1016/j. drugalcdep.2012.10.013

Rumpf, H.J., Bischof, G., Bischof, A. \& Hoch, E. (2017). Lübecker Memorandum zur Zukunft der Suchtkrankenversorgung. Suchttherapie, 18(S 01), S-32. https://doi.org/10.1055/s-0037-1 604624

Sächsische Landesstelle gegen die Suchtgefahren e.V. (2021). Sucht 2020 - Bericht der Suchthilfe in Sachsen. Dresden: Sächsische Landesstelle gegen die Suchtgefahren e.V. Verfügbar unter https://www.slsev.de/fileadmin/dokumente/allgemeines/ Sucht2020.pdf

Sächsisches Staatsministerium für Soziales und Verbraucherschutz. (Hrsg.). (2019). 3. Sächsischer Drogen- und Suchtbericht. Dresden: Sächsisches Staatsministerium für Soziales und Verbraucherschutz. Verfügbar unter https://publikationen. sachsen.de/bdb/artikel/33781
Sarkar, S., Balachander, S. \& Basu, D. (2014). Perceived harmfulness of substance use: A pilot study. Indian Journal of Community Medicine: Official Publication of Indian Association of Preventive \& Social Medicine, 39, 26-29. https://doi.org/10.4103/ 0970-0218.126350

Thal, S.B. \& Lommen, M.J.J. (2018). Current perspective on MDMA-assisted psychotherapy for posttraumatic stress disorder. Journal of Contemporary Psychotherapy, 48, 99-108. https:// doi.org/10.1007/s10879-017-9379-2

Thaller, R., Specht, S., Künzel, J. \& Braun, B. (2016). Suchthilfe in Deutschland 2016. Jahresbericht der deutschen Suchthilfestatistik (DSHS). München: IFT Institut für Therapieforschung. Verfügbar unter https://www.suchthilfestatistik.de/fileadmin/user _upload_dshs/Publikationen/Jahresberichte/DSHS_Jahresbericht_2016.pdf

Thase, M. E., Salloum, I. M. \& Cornelius, J. D. (2001). Comorbid alcoholism and depression: Treatment issues. Journal of Clinical Psychiatry, 62(Suppl 20), 32-41.

Thomasius, R., Arnaud, N., Holtmann, M. \& Kiefer, F. (2020). Substanzbezogene Störungen im Jugend- und jungen Erwachsenenalter. Zeitschrift für Kinder- und Jugend psychiatrie und Psychotherapie, 48, 448-452.

Zenker, C., Bammann, K. \& Zenker, C. (2002). Genese und Typologisierung der Abhängigkeitserkrankungen bei Frauen (Schriftenreihe des Bundesministeriums für Gesundheit und soziale Sicherung, Bd. 148). Baden-Baden: Nomos.

\section{Historie}

Manuskript eingereicht: 15.06.2021

Nach Revision angenommen: 13.11.2021

Onlineveröffentlichung: 05.01.2021

\section{Interessenkonflikte}

VR erhielt Vergütungen für Beratungs- und Schreibtätigkeiten von Lilly, Novartis und Shire Pharmaceuticals, Vortragshonorare von Lilly, Novartis, Shire Pharmaceuticals/Takeda und Medice Pharma sowie Forschungsunterstützung von Shire Pharmaceuticals/ Takeda und Novartis. Er führte (und führt derzeit) klinische Studien in Zusammenarbeit mit den Unternehmen Novartis, Shire Pharmaceuticals/Takeda, Servier und Otsuka durch. Förderungen haben weder das Forschungsthema noch die Ergebnisse oder Schlussfolgerungen der vorliegenden Arbeit beeinflusst. MW, JA, LB, YG und SKP berichten ebenfalls keine Interessenkonflikte.

\section{Förderung}

Die Studie wurde durch die Sächsische Aufbaubank - Förderbank - (SAB) gefördert (Grant 100362999 an YG).

Open-Access-Veröffentlichung ermöglicht durch die Technische Universität Dresden.

\section{Melina Wiedmann, M.Sc.}

Universitätsklinikum Carl Gustav Carus an der Technischen Universität Dresden

Anstalt des öffentlichen Rechts des Freistaates Sachsen Klinik und Poliklinik für Kinder- und Jugendpsychiatrie und -psychotherapie

Fetscherstr. 74

01307 Dresden

Deutschland

melina.wiedmann@ukdd.de 